\title{
Antimicrobial Stewardship Programs: How to Start and Steer a Successful Program
}

\author{
Richard H. Drew, PharmD, MS, BCPS
}

\begin{abstract}
BACKGROUND: Antimicrobial stewardship programs (ASPs) promote the appropriate use of antimicrobials by selecting the appropriate dose, duration, and route of administration. The appropriate use of antimicrobials has the potential to improve efficacy, reduce treatment-related costs, minimize drug-related adverse events, and limit the potential for emergence of antimicrobial resistance.
\end{abstract}

OBJECTIVE: To summarize ASP tactics that can improve the appropriate use of antimicrobials in the hospital setting. Several measures can be used to implement such programs and gain multidisciplinary support while addressing common barriers.

SUMMARY: Implementation of an ASP requires a multidisciplinary approach with an infectious diseases physician and a clinical pharmacist with infectious diseases training as its core team members. As identified by recently published guidelines, 2 proactive strategies for promoting antimicrobial stewardship include: (1) formulary restriction and pre-authorization, and (2) prospective audit with intervention and feedback. Other supplemental strategies involve education, guidelines and clinical pathways, antimicrobial order forms, de-escalation of therapy, intravenous-to-oral (IV-to-P0) switch therapy, and dose optimization. Several barriers exist to successful implementation of ASPs. These include obtaining adequate administrative support and compensation for team members. Gaining physician acceptance can also be challenging if there is a perceived loss of autonomy in clinical decision making.

CONCLUSION: ASPs have the potential to reduce antimicrobial resistance, health care costs, and drug-related adverse events while improving clinical outcomes. The efforts and expense required to implement and maintain ASPs are more than justified given their potential benefits to both the hospital and the patient.

J Manag Care Pharm. 2009;15(2)(Suppl):S18-S23

Copyright $\odot$ 2009, Academy of Managed Care Pharmacy. All rights reserved.

\section{Author}

RICHARD H. DREW, PharmD, MS, BCPS, is Professor, Campbell University School of Pharmacy, and Associate Professor of Medicine (Infectious Diseases), Duke University School of Medicine, Duke Medical Center, Durham, North Carolina.

CORRESPONDENCE: Richard H. Drew, PharmD, MS, BCPS, Box 3306, Duke Medical Center, Durham, NC 27710. Tel. 919.681.6793; Fax: 919.681.7494; E-mail: richard.drew@duke.edu
T he timely selection and administration of appropriate antimicrobial therapy can significantly impact treatment outcomes, especially in patients with severe or life-threatening infections.$^{1,2}$ In an effort to optimize antimicrobial therapy while reducing treatment-related costs, minimizing adverse events, and decreasing the risk of development of antimicrobial resistance, many institutions are implementing antimicrobial stewardship programs (ASPs).

\section{Justification for ASPs}

Though it is difficult to establish causal relationships (because multiple factors contribute to the development and persistence of antimicrobial resistance), ASPs have the potential to limit the emergence and spread of resistant pathogens. A number of observations have suggested an association between antimicrobial use and the emergence of resistance. First, in vivo selection of resistance during antimicrobial therapy can cause de novo resistance, which can quickly spread to other patients in the setting of poor infection control measures (i.e., improper hand hygiene techniques or environmental contamination). Second, patients harboring a resistant organism (when transferred to a particular unit) may introduce the resistant strain. Third, resistance genes can also be transferred between organisms to create new resistant organisms. Fourth, ASPs attempt to reduce antimicrobial pressures that have been shown to promote resistance development. $^{3-5}$ For example, several studies have reported parallel changes in antimicrobial use and the prevalence of resistance. ${ }^{6-9}$ Prior antimicrobial use is common in patients with health careassociated infections caused by resistant strains. ${ }^{10}$ Areas within hospitals with higher rates of antimicrobial resistance also tend to have higher rates of antimicrobial use. ${ }^{9}$ Increasing the duration of antimicrobials also increases the risk for colonization with resistant organisms.

Antimicrobial stewardship aims to promote the appropriate use of antimicrobials-the right selection, duration, dose, and route of administration. Promoting the appropriate use of antimicrobials is intended to improve clinical outcomes by reducing the emergence of resistance, limiting drug-related adverse events, and minimizing the risk of unintentional consequences associated with antimicrobial use (such as an increased risk of Clostridium difficile infection). , $^{3,11,12}$

ASPs also have the potential to reduce antimicrobial costs by limiting the overuse and inappropriate use of these agents and by promoting active intravenous-to-oral (IV-to-PO) switch therapy. By reducing the unnecessary use of antimicrobials, a well-designed ASP has the additional advantages of reducing (a) the risk of drug-related adverse events and their associated costs, and (b) the emergence of resistance and, hence, minimizing infections caused by resistant pathogens. Infections caused by resistant organisms are associated with poorer clinical outcomes, prolonged hospital length of stay (LOS), and higher overall costs compared to infections caused by susceptible organisms. ${ }^{13-15}$ Therefore, by promoting the appropriate use of antimicrobials, ASPs can have a broad impact on improving clinical outcomes while reducing overall health care costs. 

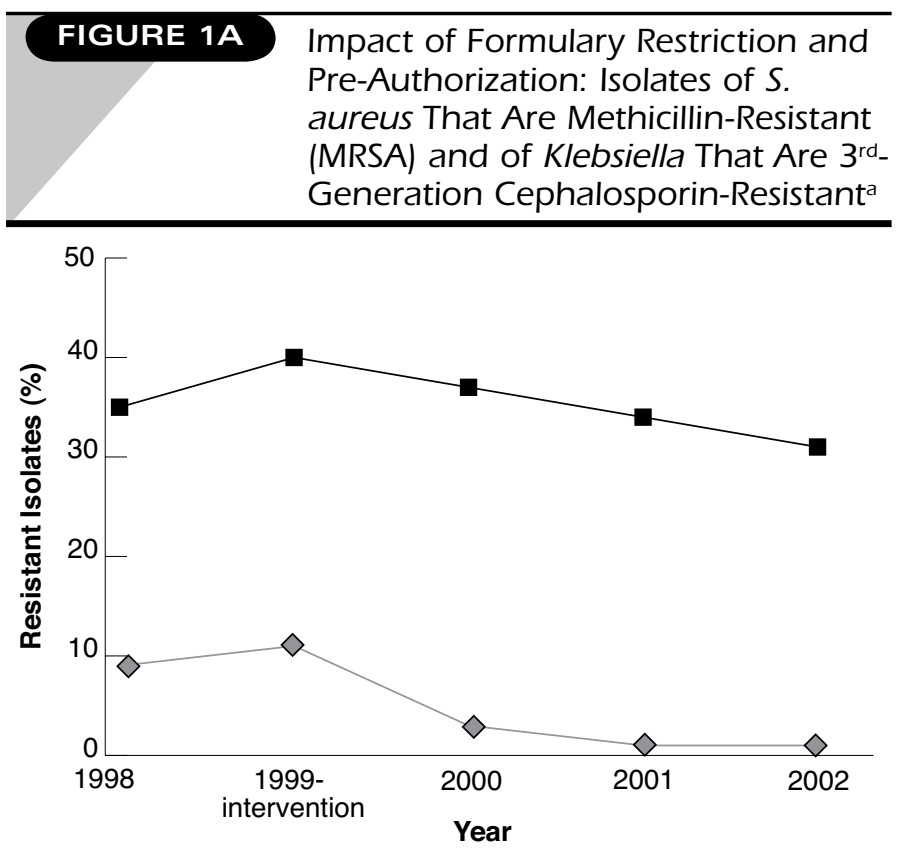

口MRSA $\diamond$ 3rd-Generation Cephalosporin-Resistant Klebsiella

\section{Stewardship Tactics}

The Infectious Diseases Society of America/Society for Healthcare Epidemiology of America (IDSA/SHEA) guidelines ${ }^{3}$ identify 2 core proactive evidence-based strategies for promoting antimicrobial stewardship: (1) formulary restriction and pre-authorization, and (2) prospective audit with intervention and feedback.

Formulary Restriction and Pre-Authorization. The strategy of formulary restriction and pre-authorization involves limiting the use of specified antimicrobials to certain approved indications. An antimicrobial committee creates guidelines pertaining to the approved use of agents. If necessary, designated personnel are made available for the approval process. The strategy leads to direct control over antimicrobial use at an institution and educational opportunities for prescribers when a request is made. The major disadvantage of this strategy is that prescribers can have a perceived loss of autonomy when making clinical decisions. Personnel also need to be available for consultation at all times. As with many ASP tactics, there is an initial cost to implement and monitor the effectiveness of such programs.

Formulary restrictions have been proven to impact antimicrobial use. ${ }^{16,17}$ One intervention at the University of Kentucky Chandler Medical Center in 1999 involved multiple aspects: (a) the removal of ceftazidime and cefotaxime from the formulary, (b) the restriction of ceftriaxone and carbapenem use to only approved indications, (c) the addition of cefepime to the formulary, (d) the replacement of ciprofloxacin with levofloxacin on the formulary, and (e) a 72-hour stop order on all vancomycin requests. ${ }^{16}$ Follow-up analysis evaluated antimicrobial use and resistance rates in selected organisms. In 2000, antimicrobial expenditures decreased by over $\$ 200,000$ (despite an increase
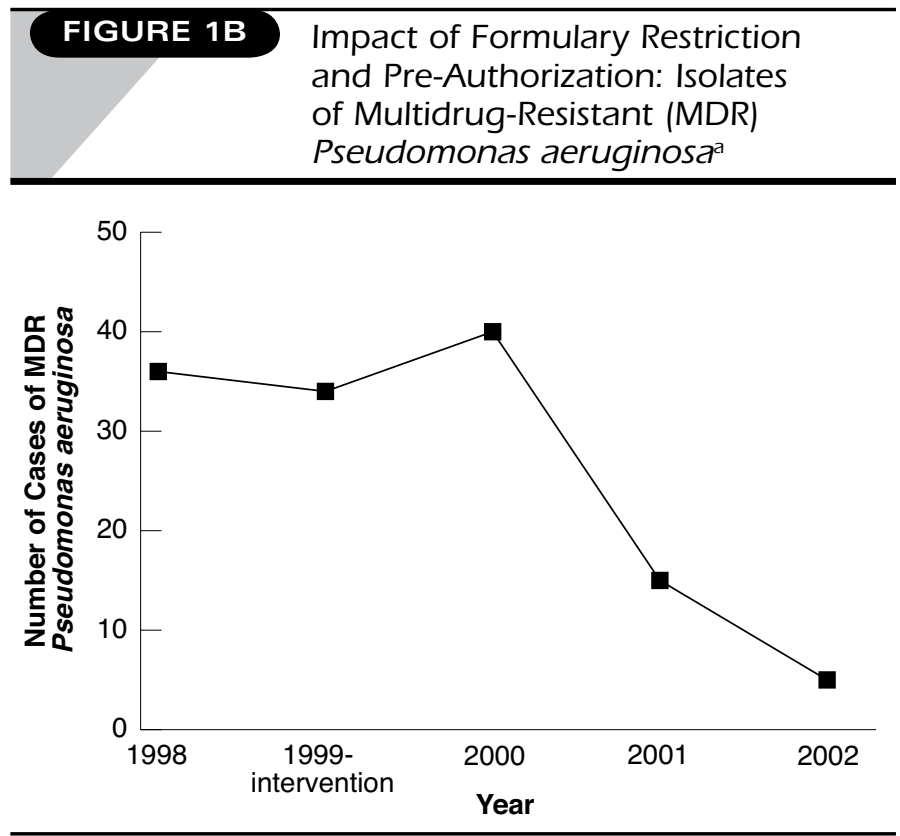

aSource: Martin et al. ${ }^{16}$

in inpatient days) and further declined by $\$ 600,000$ as of 2002 (when compared to 1998 expenditures). Not surprisingly, ceftazidime, cefotaxime, and ceftriaxone use decreased by nearly $80 \%$ by 2002. Another benefit of the ASP has been a decrease in resistance rates of several important pathogens, including multidrugresistant $P$. aeruginosa and methicillin-resistant Staphylococcus aureus (MRSA; Figures la and lb). The benefits from implementing this program have shown to be persistent.

Prospective Audit With Intervention and Feedback. A strategy of prospective audit with intervention and feedback involves a daily review of targeted agents for appropriateness. Follow-up intervention, if necessary, involves contacting the prescriber to recommend alternative agents. This tactic requires an antimicrobial committee to develop guidelines for appropriate use of targeted agents, and personnel (usually clinical pharmacists) are needed to perform the reviews and follow-up communication on a daily basis. The advantage of this strategy is that prescribers do not experience any perceived loss of autonomy, particularly if suggested changes by the reviewers are voluntary. This tactic also allows opportunity for educating prescribers through follow-up.

When utilized in a medium-sized community teaching hospital in Boston, this strategy resulted in significant reductions in inappropriate use of broad-spectrum intravenous agents, particularly third-generation cephalosporins. ${ }^{18}$ An antimicrobial management team (consisting of an infectious diseases physician and an infectious diseases-trained pharmacist) reviewed antimicrobial orders for all patients receiving parenteral thirdgeneration cephalosporins, aztreonam, parenteral fluoroquinolones, or imipenem. The recommendations of the antimicrobial management team were communicated to the prescribers via nonpermanent chart notes. Following the implementation of the program, parenteral antimicrobial use decreased steadily from 


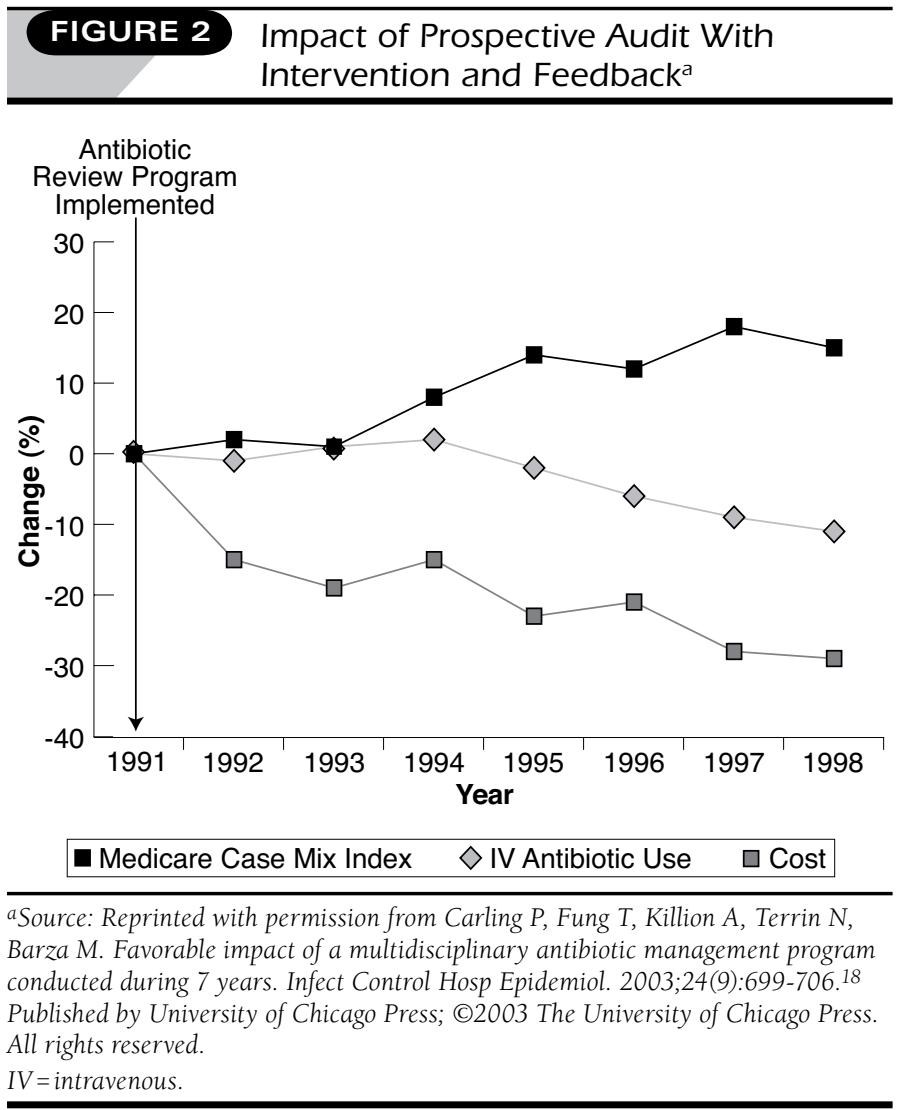

1994 to 1998 while costs of parenteral antimicrobials decreased by nearly $30 \%$ (Figure 2), despite a 15\% increase in the Medicare Case Mix Index and a $56 \%$ increase in ICU patient-days. The effect of this strategy on resistance and nosocomial infections was less clear. The rate of Clostridium difficile infection showed an initial decrease in 1993 and remained fairly steady after this (Figures $3 \mathrm{a}$ and 3b). ${ }^{18}$ Similarly, the number of infections caused by ceftazidime-resistant Enterobacteriaceae decreased following implementation of the program, followed by a steady rate until 1996 and then a decrease again in 1997 and 1998. However, vancomycin-resistant enterococci (VRE) were first isolated in 1995 and their number grew dramatically in 1996. MRSA rates did not seem to be affected by the program and grew steadily. ${ }^{18}$

Supplemental Strategies. Other supplemental strategies can also play a pivotal role in ASPs. ${ }^{3}$ These include education, guidelines and clinical pathways, antimicrobial order forms, streamlining or de-escalation, dose optimization, and IV-to-PO switch.

Education is essential for any program that is designed to influence prescribing behaviors. Programs are needed to disperse information in an accurate and timely fashion. Since personnel can change over time, it is also important that the message be repeated routinely. Effective implementation of ASPs will incorporate education along with active strategies, such as prospective audit and intervention. ${ }^{19}$

Guidelines and clinical pathways can improve antimicrobial utilization by multidisciplinary development of evidence-based guidelines that incorporate local microbiology and resistance pat-

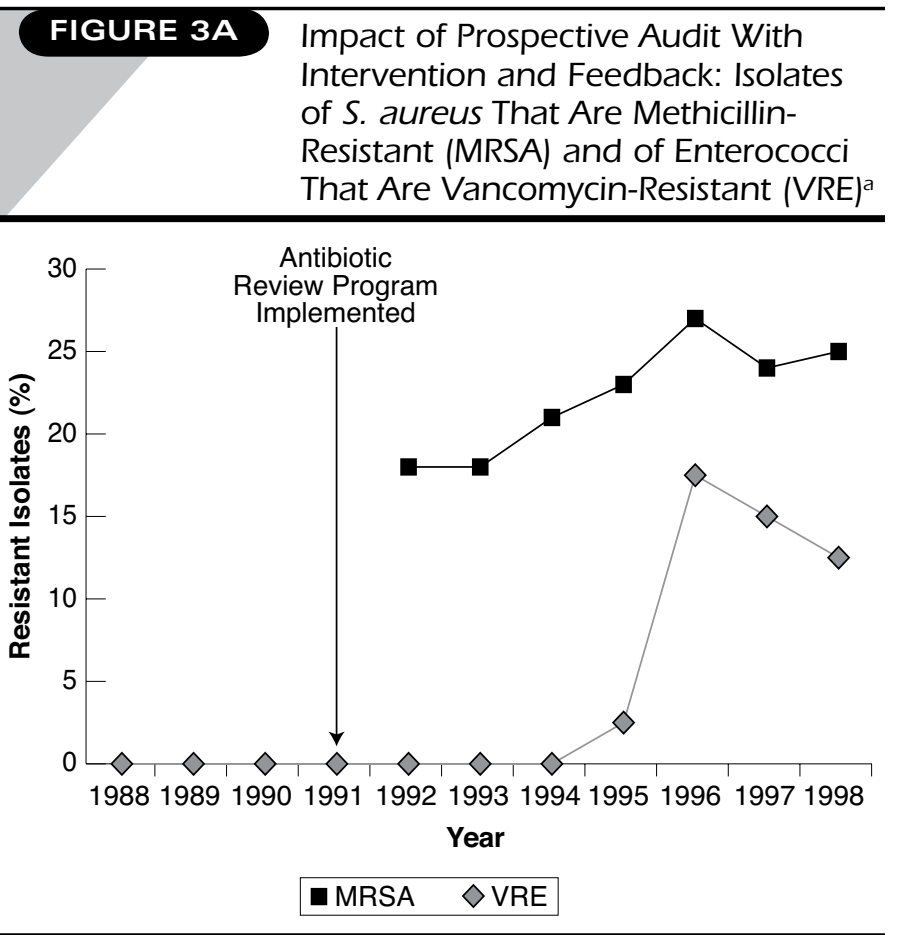

a Source: Carling et al. 18 Published by University of Chicago Press. All rights reserved.

terns. ${ }^{3}$ However, it is important to note that antimicrobial selection is only one component of these recommendations. Diagnosis and testing, admission criteria, nursing care, conversion to oral medication, and discharge planning can also impact quality of care and resource utilization. ${ }^{3}$ One study that incorporated a critical pathway at 20 hospitals for patients with CAP showed an $18 \%$ decrease in admissions for low-risk patients and significantly lower LOS and duration of IV therapy when compared to conventional therapy, resulting in significant cost savings. ${ }^{20}$

Antimicrobial order forms can be an effective tactic to decrease antimicrobial consumption by implementing automatic stop orders and/or requiring physicians to justify antimicrobial use. ${ }^{21}$ However, prescribers may view the process of filling out these forms as inconvenient and time consuming. The transition to computerized data entry systems at institutions may improve the use and convenience of such strategies.

Streamlining or de-escalation can decrease antimicrobial exposure and save costs when empiric therapy involves a combination of agents to ensure broad-spectrum coverage. Once culture results identify the pathogen, a planned removal of antimicrobials that are not necessary or that provide redundant coverage is initiated to provide more targeted therapy. For example, if vancomycin is initially included in the treatment regimen but culture results show an absence of MRSA, vancomycin can then be removed. This approach can lead to substantial cost savings without affecting clinical outcomes. ${ }^{22,23}$

Dose optimization, an important part of antimicrobial stewardship, takes into account factors such as the pharmacokinetics and pharmacodynamics of the agent, patient and pathogen 


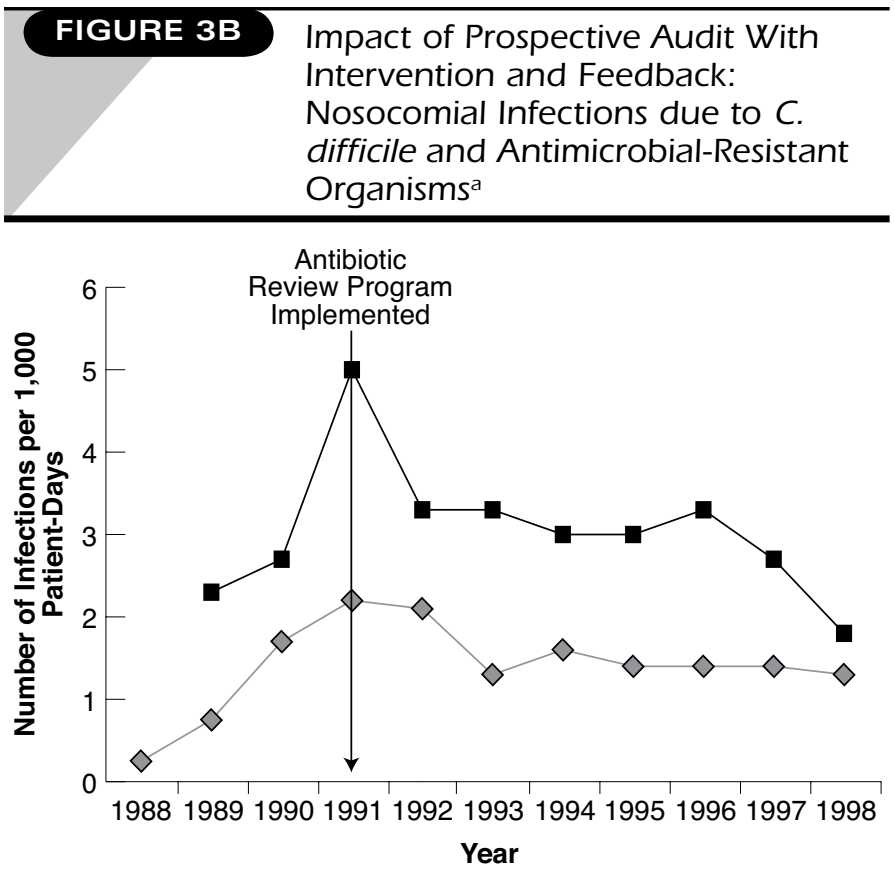

- Resistant Enteric Gram-Negative Rods $\diamond C$. difficile

a Source: Reprinted with permission from Carling P, Fung T, Killion A, Terrin N, Barza M. Favorable impact of a multidisciplinary antibiotic management program conducted during 7 years. Infect Control Hosp Epidemiol. 2003;24(9):699-706. ${ }^{18}$ Published by University of Chicago Press; (c2003 The University of Chicago Press. All rights reserved.

characteristics, and the site of infection when selecting the most appropriate antimicrobial regimen. Dose optimization strategies may include prolonged infusion of $\beta$-lactams, extended dosing intervals of aminoglycosides, or higher doses of fluoroquinolones to ensure that pharmacokinetic-pharmacodynamic targets are met.24-26

IV-to-PO switch, discussed in the article by Dr. Nicolau in this supplement, is an effective tactic to decrease the LOS and health care costs.

The role of antimicrobial cycling in antimicrobial stewardship is not clear; insufficient data are available to recommend this strategy for routine use. Antimicrobial cycling involves the deliberate scheduled removal and substitution of specific antimicrobials or classes of antimicrobials within an institution to avoid or reverse the emergence of antimicrobial resistance. ${ }^{27}$ As the scheduled antimicrobial is changed on a regular basis, adherence can be difficult with these programs mainly because prescribers may be unaware of the current scheduled antimicrobial. ${ }^{28}$

The routine use of combination therapy is not recommended given a lack of data supporting its impact on preventing resistance development or improving outcomes. ${ }^{29}$ However, empiric combination therapy can be important when treating severely ill patients to ensure early adequate coverage of potential pathogens. ${ }^{30}$ Once culture results are available, de-escalation of therapy is recommended to provide targeted therapy and reduce antimicrobial exposure. , $30^{3}$

\section{FIGURE 4 Stewardship Strategies ${ }^{a}$}

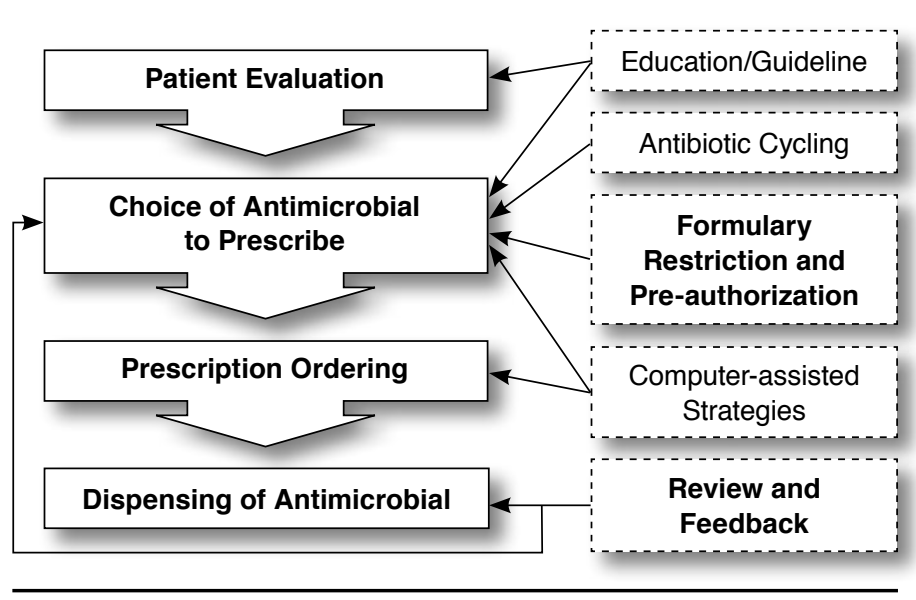

aSource: MacDougall C, Polk RE. Antimicrobial stewardship programs in health care systems. Clin Microbiol Rev. 2005;18(4):638-56. ${ }^{31}$ Reproduced with permission from American Society for Microbiology. (C2005 American Society for Microbiology. All rights reserved.

\section{Stewardship Tactics at Various Stages of Patient Management}

Stewardship tactics can be used at the various stages of managing a patient with an infectious disease (Figure 4). ${ }^{31}$ During patient evaluation, clinician education as well as management guidelines can aid in the proper diagnosis and the further actions needed (admission, laboratory testing, etc). Selecting the initial antimicrobial can also be impacted by education and the implementation of guidelines, as well as any formulary restriction and preauthorization policies. Computer-assisted strategies can be useful during the stage of antimicrobial selection, while a review and feedback strategy can help provide additional educational opportunities to the prescriber and offer a chance to adjust therapy and amend prescribing practices.

\section{Impact of ASPs}

Though more data are needed to demonstrate the benefits of the programs, ASPs have the potential to reduce resistance, health care costs, and drug-related adverse events while improving clinical outcomes. The impact of ASPs on bacterial resistance can be difficult to assess due to the multiple factors that can influence resistance development and spread. Optimized antimicrobial use is thought to help reduce the emergence of resistance, though few prospective randomized trials have attempted to analyze this. ${ }^{32}$ Other studies that have attempted to assess various strategies to minimize resistance development usually have multiple confounding variables that can make it difficult to attribute any impact to one tactic. However, as discussed earlier, given an apparent association between antimicrobial use and the emergence of resistance, ASPs that reduce the inappropriate use of antimicrobials will decrease the selection pressure for the emergence of resistance.

The IDSA/SHEA guidelines report that comprehensive programs can lead to a reduction in antimicrobial use by $22 \%-36 \%$, resulting in significant cost savings. ${ }^{3}$ The study by Martin et al., 
presented earlier, demonstrated how a policy of formulary restriction and pre-authorization can result in substantial pharmacy cost savings. ${ }^{16}$ These programs can provide substantial economic benefits irrespective of the size of the institution.

The impact of ASPs on clinical outcomes and adverse events can also be difficult to measure given the multifactorial nature of these issues. In one example of prospective audit and feedback, the rate of $C$. difficile infections decreased and remained stable after implementation of the program. ${ }^{18}$ ASPs that reduce overall antimicrobial usage by minimizing the inappropriate use of these agents will have the potential to decrease the risk of drug-related adverse events and unintended consequences.

\section{Implementing an ASP}

The rationale, design, and implementation of ASPs have been described extensively in the medical literature. $3,31,33-35$

Creating an ASP involves multiple steps. ${ }^{32}$ Baseline information should be obtained pertaining to antimicrobial use, expenditure, and institutional bacterial susceptibilities derived from the hospital antibiogram. This can help identify recurrent problems with antimicrobial use at the institution, such as overuse of a particular class or failure to switch from IV-to-PO when appropriate. An antimicrobial management strategy should be formulated, and an antimicrobial stewardship team with welldefined responsibilities formed. A multidisciplinary approach should be considered when selecting the ASP team members. The IDSA/SHEA guidelines recommend that the 2 core members of the team should include an infectious diseases physician and a clinical pharmacist with infectious diseases training. ${ }^{3}$ Other critical members of the team can include a clinical microbiologist, a hospital epidemiologist, an infection control professional, and an information system specialist.

It is important to obtain support from the hospital administration as well as build relationships within the institution to help gain acceptance of the program once implemented. The hospital administration should give core team members the authority to enforce stewardship tactics. The ASP team members should also be fairly compensated for the additional time and effort needed to implement the ASP. One survey of infectious diseases consultants identified lack of compensation as a major barrier to implementing ASPs. ${ }^{36}$ Prior to implementation of a program, the ASP team should negotiate the expected outcomes with hospital administration, which should be measurable and attainable.

Physician acceptance is extremely important during the design and implementation of an ASP. Adherence to ASPs should be monitored on a regular basis in order to identify ways in which physicians may try to circumvent ASP policies. One study described the experience at the University of Pennsylvania, where requests for restricted antimicrobials from 8:00 a.m. to 10:00 p.m. must be approved by an infectious diseases-trained pharmacist or infectious diseases fellow. ${ }^{37}$ However, outside of these active ASP hours, restricted antimicrobials may be ordered without prior approval, though all orders still require approval by the ASP for continuation of treatment. The study evaluated whether prescribers were waiting until after the approval period ended (10:00 p.m.) for ordering restricted antimicrobials. Antimicrobial orders over a 3-month period were compared from one hour before (9:00-9:59 p.m.) and one hour after (10:00-10:59 p.m.) the ASP approval period. A greater proportion of antimicrobials ordered after the ASP approval period was for restricted antimicrobials
( $57 \%$ vs. $49.9 \%, P=0.02$ ). Furthermore, once the ASP evaluated new antimicrobial orders for continuation of therapy, a significantly higher percentage of orders made after the ASP approval period was discontinued. The difference was most profound for orders originating from the surgical unit. This study suggests that physicians were more likely to wait until after the ASP approval period ended to order restricted antimicrobials without prior approval. These orders were more often found to be in conflict with guidelines or were unnecessary and hence discontinued. Finally, prescribers should receive positive feedback on a regular basis, and audits should be conducted routinely to monitor the effectiveness of the program.

\section{Barriers to ASPs}

Despite the many benefits of ASPs in improving antimicrobial use and clinical outcomes while reducing costs, several barriers exist that may hinder their implementation. Foremost is finding the appropriate personnel who are willing to devote the extra time and effort towards developing and enforcing ASPs. This barrier is further exacerbated by the fact the few clinicians receive additional compensation for the added responsibility. A survey by the Emerging Infectious Diseases Network found that only $18 \%$ of respondents were compensated for added responsibility. ${ }^{36}$ Hospital administration may be hesitant to fund such programs without a guarantee of future pharmacy savings.

Implementing tactics for an effective ASP will require funding to compensate those involved in the planning and monitoring of such programs. Further study is needed to understand the economic impact of ASPs as current reports are limited to single-center, longitudinal studies. ${ }^{17,18,38-41}$ However, these reports consistently show a decrease in antimicrobial use ranging from $22 \%$ to $36 \%$ and annual cost savings of $\$ 200,000$ to $\$ 900,000$ at both large academic medical centers ${ }^{17,18,38,39}$ and smaller community hospitals. ${ }^{40,41}$ These savings should more than offset any additional cost in implementing an ASP.

Another barrier is that ASP team members may not want to antagonize colleagues in other specialties as this can damage relationships and the potential for future consultations. This barrier may be circumvented by using a prospective audit with feedback tactic that makes any recommendation voluntary rather than mandatory and allows for educational opportunities. Other barriers for acceptance of ASPs may include a loss of physician autonomy pertaining to clinical decision making, a shortage of infectious diseases-trained pharmacists, restriction policies that can be onerous to adopt, and the continued need to assess the success of a program in order to sustain efforts.

\section{Future Direction of Antimicrobial Stewardship}

The IDSA/SHEA guidelines provide institutions with information needed when considering implementing an ASP. With more and more institutions implementing ASPs, it is anticipated that a growing number of studies will become available to better assess their impact-particularly, how the appropriate use of antimicrobials may impact the emergence of bacterial resistance. With the growing use of computerized order-entry and decision-support systems, ASPs may also become easier to implement and enforce while still providing opportunities to discuss with clinicians the appropriate use of antimicrobials. The greatest challenge may be in finding qualified personnel willing and able to direct such programs at each institution. 


\section{DISCLOSURES}

Richard H. Drew serves as a consultant to Merck, Theravance, Ortho-McNeil, and Schering-Plough. He receives research support from Schering-Plough, NeuTec, and Cubist and has received honoraria as a speaker from ScheringPlough, Ortho-McNeil, Enzon, sanofi-aventis, Wyeth-Ayerst, and Astellas Pharma. Drew is on the Development Team for CustomID.

Marco P. Cicero, PhD, of Vemco MedEd, LLC, contributed medical writing and editorial assistance. This article is being published as part of a supplement to the START continuing education program for pharmacists and physicians. It is supported by an educational grant from Schering-Plough Corporation.

\section{REFERENCES}

1. Kollef KE, Schramm GE, Wills AR, Reichley RM, Micek ST, Kollef MH. Predictors of 30-day mortality and hospital costs in patients with ventilatorassociated pneumonia attributed to potentially antibiotic-resistant gramnegative bacteria. Chest. 2008;134(2):281-87.

2. Kollef MH. Appropriate empiric antimicrobial therapy of nosocomial pneumonia: the role of the carbapenems. Respir Care. 2004:49(12):1530-41. 3. Dellit TH, Owens RC, McGowan JE, Jr., et al. Infectious Diseases Society of America and the Society for Healthcare Epidemiology of America guidelines for developing an institutional program to enhance antimicrobial stewardship. Clin Infect Dis. 2007;44(2):159-77.

4. Shlaes DM, Gerding DN, John JF, Jr., et al. Society for Healthcare Epidemiology of America and Infectious Diseases Society of America Joint Committee on the Prevention of Antimicrobial Resistance: guidelines for the prevention of antimicrobial resistance in hospitals. Clin Infect Dis. 1997;25(3):584-99.

5. Monroe S, Polk R. Antimicrobial use and bacterial resistance. Curr Opin Microbiol. 2000;3(5):496-501.

6. Courcol RJ, Pinkas M, Martin GR. A seven year survey of antibiotic susceptibility and its relationship with usage. J Antimicrob Chemother. 1989;23(3):441-51.

7. Conus P. Francioli P. Relationship between ceftriaxone use and resistance of Enterobacter species. J Clin Pharm Ther. 1992;17(5):303-05.

8. Polk RE, Johnson CK, McClish D, Wenzel RP, Edmond MB. Predicting hospital rates of fluoroquinolone-resistant Pseudomonas aeruginosa from fluoroquinolone use in US hospitals and their surrounding communities. Clin Infect Dis. 2004:39(4):497-503.

9. Muller AA, Mauny F, Bertin M, et al. Relationship between spread of methicillin-resistant Staphylococcus aureus and antimicrobial use in a French university hospital. Clin Infect Dis. 2003;36(8):971-78.

10. Vanderkooi OG, Low DE, Green K, Powis JE, McGeer A. Predicting antimicrobial resistance in invasive pneumococcal infections. Clin Infect Dis 2005;40(9):1288-97.

11. Lawrence SJ, Puzniak LA, Shadel BN, Gillespie KN, Kollef MH, Mundy LM. Clostridium difficile in the intensive care unit: epidemiology, costs, and colonization pressure. Infect Control Hosp Epidemiol. 2007;28(2):123-30.

12. Owens RC, Jr., Donskey CJ, Gaynes RP, Loo VG, Muto CA.

Antimicrobial-associated risk factors for Clostridium difficile infection. Clin Infect Dis. 2008;46(Suppl 1):S19-S31.

13. Combes A, Luyt CE, Fagon JY, et al. Impact of methicillin resistance on outcome of Staphylococcus aureus ventilator-associated pneumonia. Am J Respir Crit Care Med. 2004;170(7):786-92

14. Lee SY, Kotapati S, Kuti JL, Nightingale CH, Nicolau DP. Impact of extended-spectrum beta-lactamase-producing Escherichia coli and Klebsiella species on clinical outcomes and hospital costs: a matched cohort study. Infect Control Hosp Epidemiol. 2006;27(11):1226-32.

15. John JF, Jr., Fishman NO. Programmatic role of the infectious diseases physician in controlling antimicrobial costs in the hospital. Clin Infect Dis. 1997;24(3):471-85.

16. Martin C, Ofotokun I, Rapp R, et al. Results of an antimicrobial control program at a university hospital. Am J Health Syst Pharm. 2005;62(7):732-38

17. Rapp RP, Evans ME, Martin C, Ofotokum I, Empey KL, Armitstead JA. Drug costs and bacterial susceptibility after implementing a singlefluoroquinolone use policy at a university hospital. Curr Med Res Opin. 2004;20(4):469-76

18. Carling P, Fung T, Killion A, Terrin N, Barza M. Favorable impact of a multidisciplinary antibiotic management program conducted during 7 years. Infect Control Hosp Epidemiol. 2003;24(9):699-706.

19. Bantar C, Sartori B, Vesco E, et al. A hospitalwide intervention program to optimize the quality of antibiotic use: impact on prescribing practice, antibiotic consumption, cost savings, and bacterial resistance. Clin Infect Dis. 2003;37(2):180-86
20. Marrie TJ, Lau CY, Wheeler SL, Wong CJ, Vandervoort MK, Feagan BG; CAPITAL Study Investigators. A controlled trial of a critical pathway for treatment of community-acquired pneumonia: CommunityAcquired Pneumonia Intervention Trial Assessing Levofloxacin. JAMA. 2000;283(6):749-55.

21. Echols RM, Kowalsky SF. The use of an antibiotic order form for antibiotic utilization review: influence on physicians' prescribing patterns. J Infect Dis. 1984;150(6):803-07.

22. Glowacki RC, Schwartz DN, Itokazu GS, Wisniewski MF, Kieszkowski $\mathrm{P}$, Weinstein RA. Antibiotic combinations with redundant antimicrobial spectra: clinical epidemiology and pilot intervention of computer-assisted surveillance. Clin Infect Dis. 2003;37(1):59-64.

23. Soo Hoo GW, Wen YE, Nguyen TV, Goetz MB. Impact of clinical guidelines in the management of severe hospital-acquired pneumonia. Chest. 2005;128(4):2778-87.

24. Dunbar LM, Wunderink RG, Habib MP, et al. High-dose, short-course levofloxacin for community-acquired pneumonia: a new treatment paradigm. Clin Infect Dis. 2003;37(6):752-60.

25. Nicolau DP. Pharmacodynamic optimization of beta-lactams in the patient care setting. Crit Care. 2008;12(Suppl 4):S2.

26. Nicolau DP, Freeman CD, Belliveau PP, Nightingale CH, Ross JW, Quintiliani R. Experience with a once-daily aminoglycoside program administered to 2,184 adult patients. Antimicrob Agents Chemother 1995;39(3):650-55.

27. Fridkin SK. Routine cycling of antimicrobial agents as an infectioncontrol measure. Clin Infect Dis. 2003;36(11):1438-44.

28. Martinez JA, Nicolas JM, Marco F, et al. Comparison of antimicrobial cycling and mixing strategies in two medical intensive care units. Crit Care Med. 2006;34(2):329-36.

29. Klibanov OM, Raasch RH, Rublein JC. Single versus combined antibiotic therapy for gram-negative infections. Ann Pharmacother. 2004;38(2):332-37. 30. American Thoracic Society. Guidelines for the management of adults with hospital-acquired, ventilator-associated, and healthcare-associated pneumonia. Am J Respir Crit Care Med. 2005;171(4):388-416. Available at: http://ajrccm.atsjournals.org/cgi/reprint/171/4/388. Accessed February 15, 2009.

31. MacDougall C, Polk RE. Antimicrobial stewardship programs in health care systems. Clin Microbiol Rev. 2005;18(4):638-56.

32. Paskovaty A, Pflomm JM, Myke N, Seo SK. A multidisciplinary approach to antimicrobial stewardship: evolution into the 21 st century. Int J Antimicrob Agents. 2005;25(1):1-10

33. Fishman N. Antimicrobial stewardship. Am J Med. 2006;119(6 Suppl 1):S53-S61; discussion S62-S70.

34. Paterson DL. The role of antimicrobial management programs in optimizing antibiotic prescribing within hospitals. Clin Infect Dis. 2006;42(Suppl 2): $590-\mathrm{S} 95$

35. Owens RC, Jr., Fraser GL, Stogsdill P, Society of Infectious Diseases Pharmacists. Antimicrobial stewardship programs as a means to optimize antimicrobial use: insights from the Society of Infectious Diseases Pharmacists. Pharmacotherapy. 2004;24(7):896-908.

36. Sunenshine RH, Liedtke LA, Jernigan DB, Strausbaugh LJ, Infectious Diseases Society of America Emerging Infections Network. Role of infectious diseases consultants in management of antimicrobial use in hospitals. Clin Infect Dis. 2004;38(7):934-38.

37. LaRosa LA, Fishman NO, Lautenbach E, Koppel RJ, Morales KH, Linkin DR. Evaluation of antimicrobial therapy orders circumventing an antimicrobial stewardship program: investigating the strategy of "stealth dosing." Infect Control Hosp Epidemiol. 2007;28(5):551-56.

38. Ansari F, Gray K, Nathwani D, et al. Outcomes of an intervention to improve hospital antibiotic prescribing: interrupted time-series with segmented regression analysis. J Antimicrob Chemother. 2003;52(5):842-48. 39. Lutters M, Harbarth S, Janssens J-P, et al. Effect of a comprehensive, multidisciplinary, educational program on the use of antibiotics in a geriatric university hospital. J Am Geriatr Soc. 2004;52(1):112-16.

40. LaRocco A, Jr. Concurrent antibiotic review programs: a role for infectious diseases specialists at small community hospitals. Clin Infect Dis. 2003;37(5):742-43.

41. Rüttimann S, Keck B, Harmeier C, et al. Long-term antibiotic cost savings from a comprehensive intervention program in a medical department of a university-affiliated teaching hospital. Clin Infect Dis. 2004;38(3):348-56. 